\title{
Physics Based Modeling and Rendering of Vegetation in the Thermal Infrared
}

\author{
J. A. Smith \\ Laboratory for Terrestrial Physics \\ NASA Goddard Space Flight Center \\ Greenbelt, MD 20771 \\ jasmith@hemlock.gsfc.nasa.gov
}

\author{
J. R. Ballard, Jr. \\ Environmental Laboratory \\ USAE Waterways Experiment Station \\ Vicksburg, MS 39180 \\ ballard@orion.wes.army.mil
}

\begin{abstract}
We outline a procedure for rendering physically-based thermal infrared images of simple vegetation scenes. Our approach incorporates the biophysical processes that affect the temperature distribution of the elements within a scene. Computer graphics plays a key role in two respects. First, in computing the distribution of scene shaded and sunlit facets and, second, in the final image rendering once the temperatures of all the elements in the scene have been computed.

We illustrate our approach for a simple corn scene where the three-dimensional geometry is constructed based on measured morphological attributes of the row crop. Statistical methods are used to construct a representation of the scene in agreement with the measured characteristics.

Our results are quite good. The rendered images exhibit realistic behavior in directional properties as a function of view and sun angle. The root-mean-square error in measured versus predicted brightness temperatures for the scene was $2.1 \mathrm{deg} C$.
\end{abstract}

\section{Introduction}

Remote sensing requires the interpretation of measured sensor response to extract information about earth surface features. We can use remote sensing to monitor biogeophysical attributes and assess environmental conditions. The interpretation of such signals, however, is a complex process dependent on many factors including sensor response function, atmospheric state, and the interplay of the probing electromagnetic radiation (passive or active) with the underlying scene.

Models play a strategic role in developing information extraction algorithms or in sensor design studies. Traditionally, these models have been one-dimensional, plane parallel abstractions. These have proven useful for the current, large-scale global monitoring satellite systems such as the NOAA Advanced Very High Resolution Radiometer with its 1 kilometer instantaneous field-of-view. However, with the next generation, high spatial resolution satellite systems, more attention must be focused on the three-dimensional structure of underlying scenes. The new satellite systems have spatial resolutions of 1 to 30 meters which is comparable to many intrinsic scene characteristics, e.g. crop crow structure.

Remote sensing researchers have already begun to draw upon traditional computer graphics techniques to aid them in their modeling of more complex scenes, particularly in the optical reflective regime. Borel, et al. [1] and Goel, et al. [3] used the radiosity method developed by Greenberg and associates [4] to model the multi-spectral reflective response of three-dimensional plant canopies. Smith, et al. [9] also employed a statistical version of the radiosity method to compute short-wave absorption of a layered canopy for subsequent use in a one-dimensional thermal infrared model.

While progress has been made in the modeling of the infrared response of three-dimensional scenes $[6,7]$, the modeling of the thermal infrared behavior of threedimensional vegetation canopies is still in the beginning phases [8].

In this paper, we discuss a novel technique for combining computer graphics, e.g. ray tracing, with physics-based energy budget modeling to predict the thermal infrared exitance of a plant canopy. We illustrate the method for an agricultural scene where meteorological, crop morphologi$\mathrm{cal}$, and thermal infrared measurements were available.

\section{Method}

Figure 1 gives a flow chart of the calculation steps required in our approach. First, we generate the threedimensional geometry of the scene using simple measurements of the canopy including crop row spacing, height, leaf slope distribution, and a measure of the density of leaf area per unit ground area, the Leaf Area Index (LAI). The details are described below. 
We then use ray tracing to determine all of the elements in the scene which are either directly illuminated, i.e., sunlit, or in shade. For each of these sunlit or shaded elements, we separately run a thermal model to calculate their energy budget and to solve for the corresponding leaf or soil temperatures. We subsequently, again, use ray tracing to render the thermal infrared scene by tracing the thermal infrared flux, emitted by each element at its estimated temperature, which is actually received by a sensor at a specified view direction. The thermal model is driven by local meteorological conditions including local wind speed, relative humidity, air temperature, and short-wave illumination and requires only simple material properties.

We used a nested approach to simulate the geometry of the scene based on measured canopy parameters. We modeled a sample plot of $19 \mathrm{~m}$ by $19 \mathrm{~m}$ for which canopy measurements, meteorology, and thermal infrared measurements were available. We first generated twenty-five unique $19 \mathrm{~m}$ rows with a canopy width of $0.46 \mathrm{~m}$, height of $0.8 \mathrm{~m}$, total row spacing of $0.76 \mathrm{~m}$ including foliage and gap between rows, and a single sided leaf area index of 3.0 corresponding to the measured row spacing, height, and LAI. A spherical leaf slope distribution and uniform leaf azimuth distribution were used to assign orientations to the individual leaf facets. Our primitive leaf facet size was $5 \mathrm{~cm}$ by 5 $\mathrm{cm}$ providing a leaf area of $0.0025 \mathrm{~m}^{2}$. Thus, 10,488 leaf facets were required to match the measured LAI of 3.0 distributed over the $19 \mathrm{~m}$ by $0.46 \mathrm{~m}$ row area. The twenty-five rows were instanced together at a row spacing of $0.3 \mathrm{~m}$ to simulate a corn foliage plot measuring $19 \mathrm{~m}$ by $19 \mathrm{~m}$ containing approximately 262,200 facets.

Figure 2 is a visual simulation of a hemispherical picture of the scene. The sun was $73.2 \mathrm{deg}$ above the horizon and

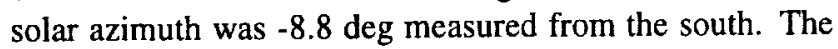
sunlit and shaded components of both canopy and soil elements are apparent. From such images, we can compute a shade mask for all elements within the scene in order to apply the appropriate energy balance calculations discussed below.

Individual leaf energy budgets are calculated as follows in terms of net radiation, $R_{n}$, sensible heat, $\mathrm{H}$, and latent heat, LE, i.e.

$$
R_{n}=L E+H
$$

A standard resistance formulation [2] is used for the calculation of latent and sensible heat in terms of the unknown leaf temperature, $T_{v}$, i.e.

$$
\begin{aligned}
H_{v} & =\frac{\left(T_{v}-T_{a}\right)}{r_{b}} \rho c_{p} \\
L E_{v} & =\left[\frac{h_{v e g} e^{*}\left(T_{v}\right)-e_{a}}{r_{c}+r_{b}}\right] \frac{\rho c_{p}}{\gamma}
\end{aligned}
$$

where $T_{a}$ is the air temperature, $h_{v e g}$ is the relative humidity of the vegetation air space, $e^{*}$ is the saturation water vapor pressure at canopy temperature $T_{v}, e_{a}$ the vapor pressure of air at air temperature $T_{a}, \rho$ is the air density, $c_{p}$ the air specific heat, and $\gamma$ the psychromatic constant. Finally, $r_{b}$ is the canopy boundary layer resistance and $r_{c}$ is the bulk stomatal resistance. Canopy stomatal resistance and the boundary layer resistance depend upon meteorological driving variables.

Net radiation to a surface, $R_{n}$, is computed as the sum of the absorbed long-wave and short-wave radiation minus the outgoing long-wave emitted thermal infrared flux. Separate calculations are required for sunlit versus shaded surfaces. For sunlit leaves, the absorbed shortwave flux is highly dependent on leaf inclination angle or the angle between the leaf and the incident solar direction. Long-wave emission is a function of the unknown leaf temperature and is given by the Stefan-Boltzmann relation.

Thus, a system of non-linear energy budget equations is solved for individual leaf temperatures for all possible leaf orientations corresponding to varying exposure of the individual leaves to direct solar flux. For sunlit leaves, one degree increments are used for leaf inclination classes. For the case simulated, sunlit leaf temperatures temperatures range from 24 to $32 \mathrm{deg} C$ with warmer temperatures occurring more frequently. For shaded leaves, the average leaf temperature was $26.5 \mathrm{deg}$ C. Average soil sunlit and shaded temperatures corresponding to the measured meteorological conditions were 55 and $46 \mathrm{deg} C$ respectively.

For the ray-tracing and rendering calculations we employed the RADIANCE program developed by Ward [10]. Small modifications were required to handle the emission from both sides of the leaf facets.

\section{Results}

Figure 3 displays a simulated thermal infrared corn image corresponding to Figure 2 rendered at one-quarter meter pixel size. High LAI or dense vegetation yields cooler temperatures in the thermal image compared to the warmer soil facets which are primarily visible looking straight down into the image.

The brightness temperature of a surface is defined as the equivalent black-body temperature of the surface, i.e. a surface with emissivity 1.0, that would yield the same total outgoing long-wave flux as the measured surface. Ground measurements of brightness temperature and meteorological driving variables were available for the $19 \mathrm{~m}$ by $19 \mathrm{~m}$ sample area. As a check on our energy budget formulations, we calculated net radiation for this area using Equations (1) and (2) and simulated the three-dimensional thermal infrared response for the test plot corresponding to 600 , $800,1000,1200,1400,1600,1800$, and 2000 hours. Figure 4 shows a comparison of the measured versus predicted brightness temperatures for these 8 time periods as calcu- 
lated from the hemispherically averaged outgoing thermal infrared flux, assuming an emissivity of 1.0. The rootmean-square error was 2.1 degrees $C$ with an $r^{2}$ of 0.93 . The error bars correspond to the estimated confidence intervals from the regression line fit to the predicted versus measured values.

\section{Summary and Conclusions}

In this paper we have given a general outline for rendering thermal infrared images of simple vegetation scenes based on physical principles. Our approach incorporates the biophysical processes that affect the temperature distribution of the vegetation elements within a scene. The thermal model is driven by readily available, standard meteorological measurements including local wind speed, relative humidity, air temperature, and short-wave illumination. Computer graphics plays a key role in two respects. First, in computing the distribution of shaded and sunlit facets within the scene and, second, in the final image rendering once the temperatures of all the elements in the scene have been computed.

We illustrated our approach for a simple corn scene where the three-dimensional geometry was constructed based on measured macroscopic morphological attributes of the row crop-viz crop height, row spacing, leaf-area-index, etc. Statistical methods were then used to randomly assign individual leaf facets with the proper orientations within rows and to construct a representation of the scene in agreement with the measured characteristics.

Our results are quite good. The rendered images exhibited realistic behavior in the directional properties as a function of view and sun angle [8]. The root-mean-square error in measured versus predicted brightness temperatures for the scene was $2.1 \mathrm{deg} C$.

Nevertheless, there are several limitations in our method. First, our vegetation scene only consisted of leaves with no woody material, e.g. trunks. The time constant for individual leaves to reach thermal equilibrium is less than 30 seconds and our equilibrium energy-budget approach was thus warranted. In soil, we used a simple trick based on results reported in the literature, i.e. modeling conduction as a fraction of net radiation (possibly with time lag). In forests, however, the woody material, e.g. trunks and branches, must be accounted for. It is more challenging to incorporate the geometry of these elements. We are experimenting with Lindenmayer systems [5].

Finally, we imposed a static wind field on the scene, consistent with average wind speed measurements and a logarithmic height profile. More realistic modeling of high frequency phenomena, e.g. leaf flutter, opens up the pandora's box of turbulence, a subject in its own right. We are investigating all of these effects and further work is clearly warranted.

\section{Acknowledgments}

The research described in this paper was supported by the Ecological Processes and Modeling Program at NASA Headquarters. Assistance was also provided by the U. S. Army Corps of Engineers Waterways Experiment Station, Research, Development, Testing and Evaluation Program.

\section{References}

[1] C. C. Borel, S. A. W. Gerstl, and B. J. Powers. The radiosity method in optical remote sensing of structured 3-D surfaces. Remote Sens. Environ., 36:13-44, 1991.

[2] D. M. Gates. Biophysical Ecology. Springer-Verlag, New York, 1980.

[3] N. S. Goel, I. Rozehnal, and R. L. Thompson. A computer graphics based model for scattering from objects of arbitrary shapes in the optical region. Remote Sens. Environ., 36:73104, 1991.

[4] D. P. Greenberg, M. F. Cohen, and K. E. Torrance. Radiosity: A method for computing global illumination. Visual Comput., 2:291-297, 1986.

[5] P. Prusinkiewicz and A. Lindenmayer. The Algorithmic Beauty of Plants. Springer-Verlag, New York, 1996.

[6] H. E. Rushmeier and S. D. Tynor. Incorporating the BRDF into an Infrared Scene Generation System. Proccedings of the SPIE, 1311, 1990.

[7] J. R. Schott, R. Raqueno, and C. Salvaggio. Incorporation of time-dependent thermodynamic model and a radiation propagation model into infrared three-dimensional synthetic image generation. Opt. Eng., 31(7):1505-1516, 1992.

[8] J. A. Smith, J. R. Ballard, and J. A. Pedelty. Effect of three-dimensional canopy architecture on thermal infrared exitance. Opt. Eng., 36:3093-3100, 1997.

[9] J. A. Smith and S. M. Goltz. Updated thermal model using simplified short-wave radiosity calculations. Remote Sens. Environ., 47:167-175, 1994.

[10] G. J. Ward and R. Shakespeare. Rendering with RADIANCE The Art and Science of Lighting Visualization. Morgan Kaufmann Publishers, San Francisco, 1997. 


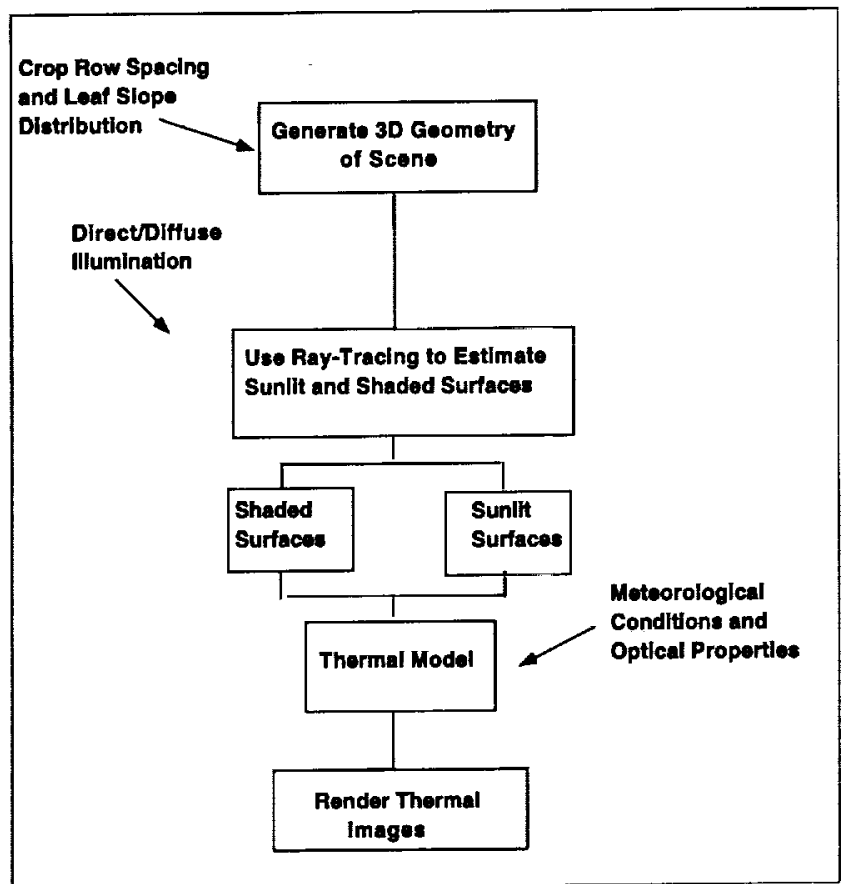

Figure 1. Flow chart of model calculations

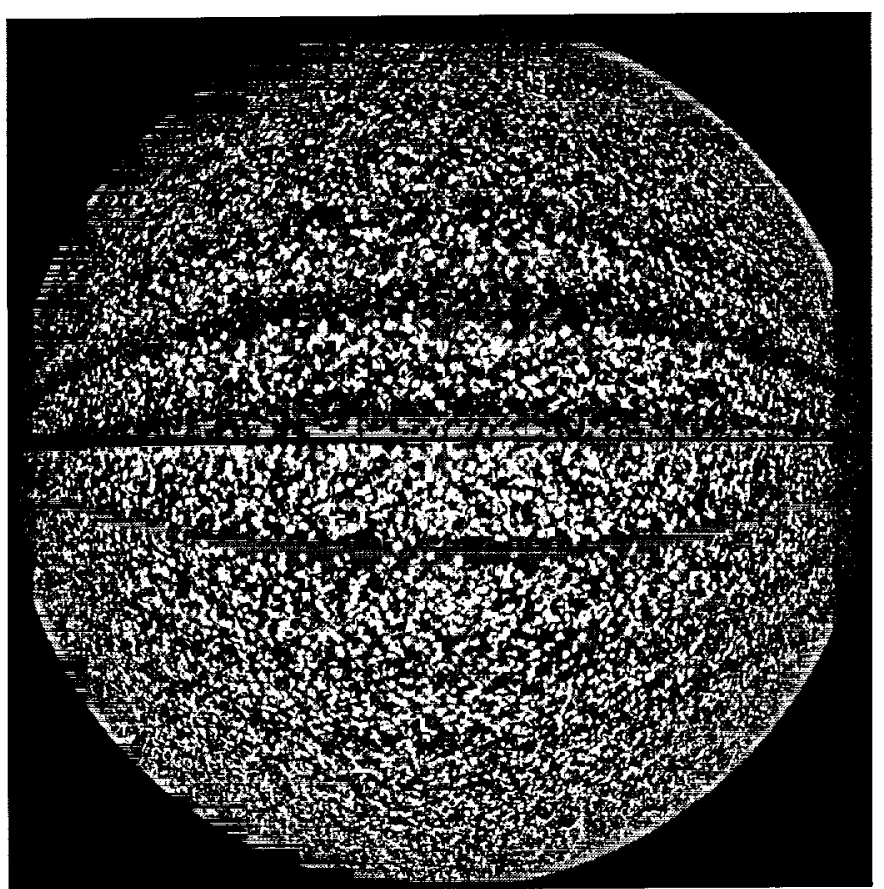

Figure 2. Simulated hemispherical visual image 


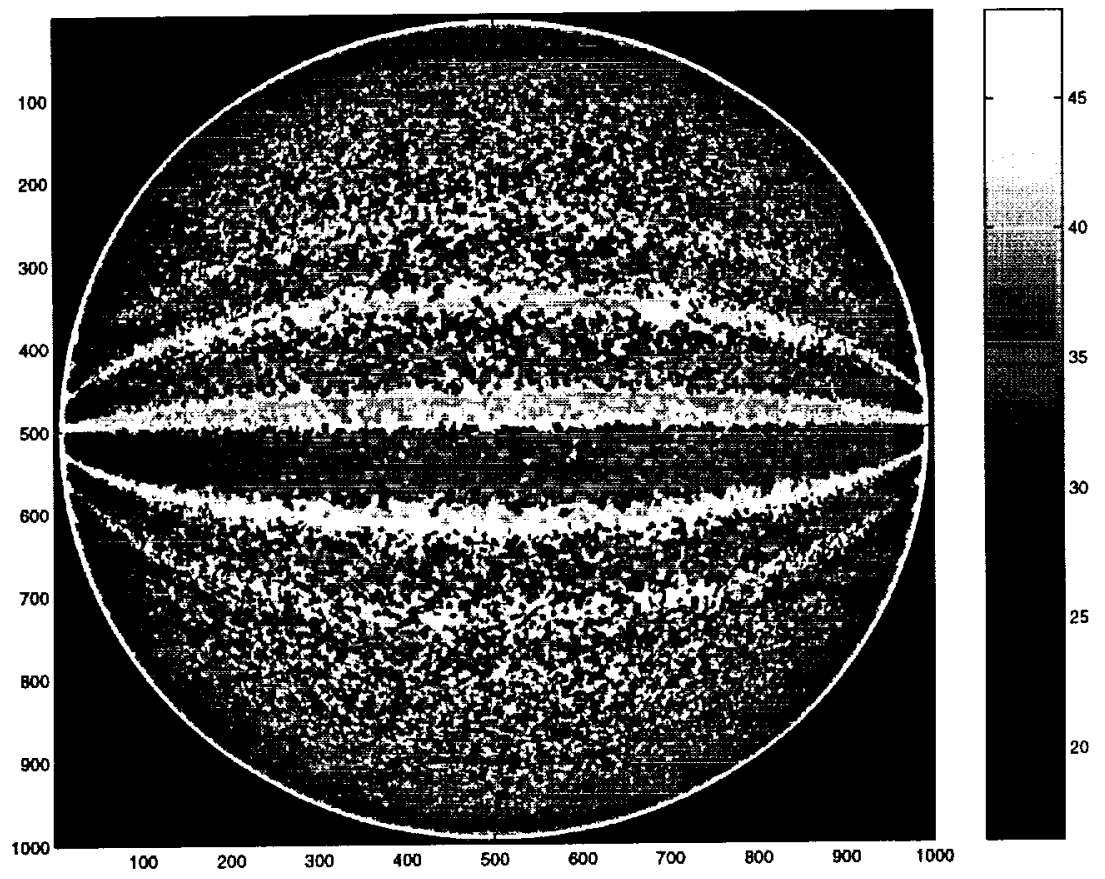

Figure 3. Simulated hemispherical thermal infared image

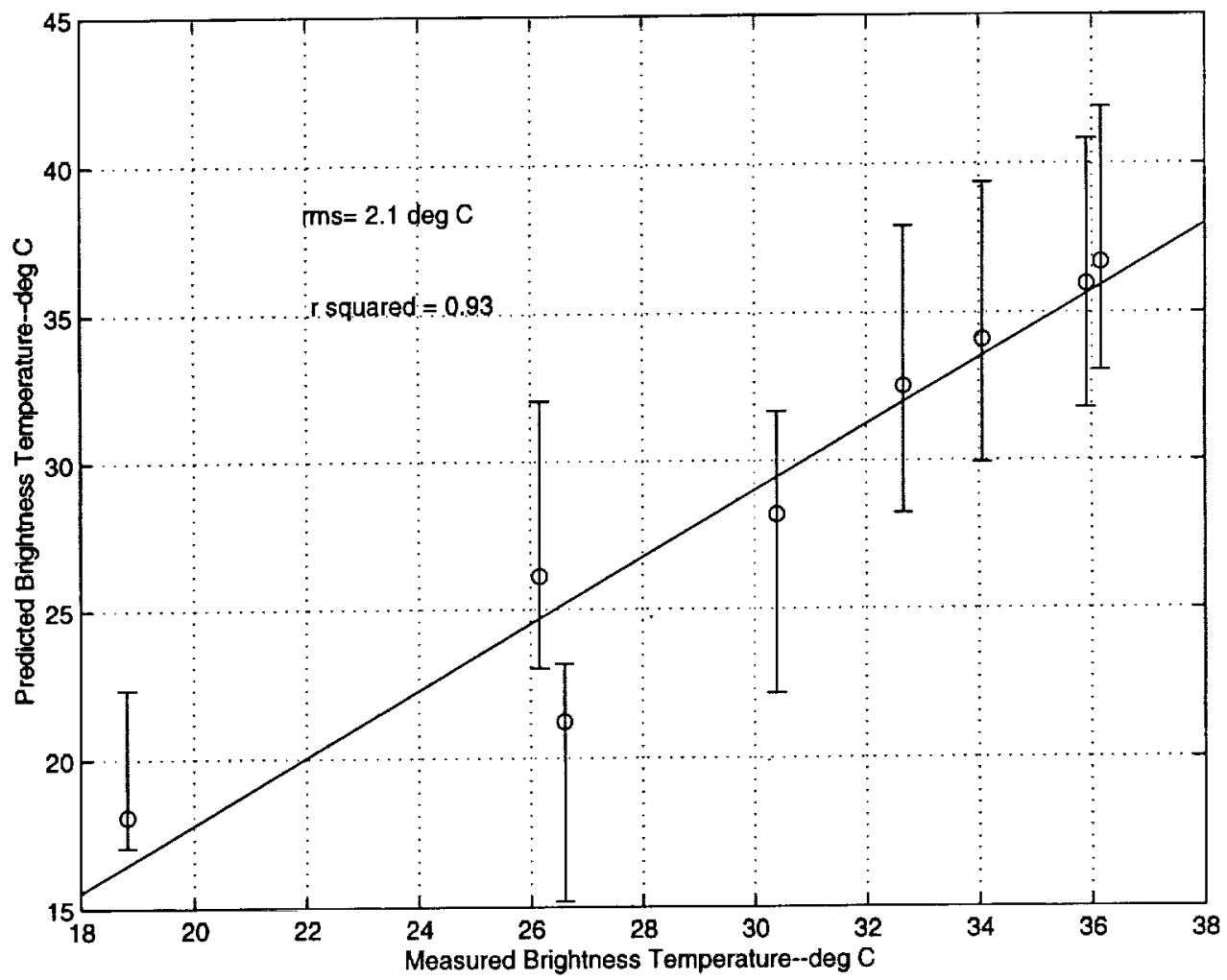

Figure 4. Comparison of predicted versus measured canopy brightness temperature 